\title{
Model Selection and Estimation of Long-Memory Time-Series Models
}

\author{
Katelijne A.E. Carbonez \\ Department of Accountancy, Finance and Insurance \\ Katholieke Universiteit Leuven \\ Naamsestraat 69-B3500, 3000 Leuven, Belgium \\ katelijne.carbonez@econ.kuleuven.be
}

February 26, 2008 


\begin{abstract}
An exploratory estimation of $\operatorname{ARFIMA}(p, d, q)$ models on agricultural spot and futures markets showed us that the estimated $d$ is quite sensitive to the number of lags included in the short-term dynamics. AIC and SIC agreed there were many lags but, familiarly, disagreed on how many. To address this issue, I run a series of Monte Carlo experiments and test the performance (i) of the AIC and the SIC in selecting $p$ and $q$ and (ii) of the AIC, the SIC and the multimodel-inference approach of Burnham and Anderson (2002) in estimating $d$. I contribute to the literature by studying highorder data generating processes-up to $(8, d, 8)$ rather than $(2, d, 0)$; by testing also the MMI-approach; and by studying the impact of excluding models close to the data generating process from the set of candidate models. Three findings stand out. First, the familiar result that, in terms of order selection, the SIC outperforms the AIC for low-order models gets reversed for high-order models. Second, for inference on the presence or absence of fractional integration, I find that the SIC still dominates both the AIC and the MMI-approach. Third, set-up snooping (if the true model is also a candidate model) has little impact.
\end{abstract}




\section{Introduction}

An adequate specification of the behavior of economic time series is paramount to the estimation, inference and prediction of many models. Both the type of model (e.g. ARMA versus ARFIMA) and the order of the model (defined here as the number of lags included) have an impact on estimation and inference.

The last few decades, a consensus has emerged that agricultural spot and futures prices do not follow simple random walks. Harvest patterns, inventory holdings, the option to substitute crops or decrease/increase acreage and technological change lead to price behavior models that exhibit mean reversion, long-term dependence, structural breaks and jumps. In this paper, I restrict my attention to the issue of long-term dependence and leave the issue of jumps and structural breaks for subsequent research.

Fractional integration has been the focus of extensive theoretical and empirical research in recent years. The main theoretic contributions stem from Granger (1980), Granger and Joyeux (1980), Hosking (1981), Geweke and Porter-Hudak (1983), Lo (1991), Sowell (1992a,b), Cheung and Diebold (1994), Robinson (1995a,b), Engle and Smith (1999), Diebold and Inoue (2001), Breitung and Hassler (2002) and Ditman and Granger (2002) . The empirical contributions span different areas of economics, not only agricultural research. For early research, we refer to the excellent survey paper of Baillie (1996). More recent research includes studies by Bollerslev and Mikkelsen (1996), Hyung and Franses (2001), Bos et al (2002), Jin and Frechette (2004) and Bhardwaj and Swanson (2006).

The empirical analysis of long-memory $\operatorname{ARFIMA}(p, d, q)$ processes usually assumes that the short-term dynamics of the data, the conventional autoregressive $(p)$ and moving average $(q)$ parameters, are given. Most frequently, $p$ and $q$ are set to zero 
or one. Real world data however may have a better fit with higher orders of $p$ and $q$. Though extensive research has been conducted concerning order selection for shortmemory $\operatorname{ARMA}(p, q)$ processes, few papers have analyzed the problem of order selection for long-memory processes. Bollerslev and Mikkelsen (1996) briefly discuss the performance of the Akaike information criterion (AIC) and the Schwartz information criterion (SIC) to discriminate between $\operatorname{FIGARCH}(1, d, 0)$ and $\operatorname{GARCH}(1,0)$ models. Both criteria seem to perform relatively well in that context. Beran, Bhansali and Ocker (1998) examine model and order selection for long-memory autoregressive processes $(\operatorname{ARFIMA}(p, d, 0))$ with $p$ zero, one or two and show that the SIC outperforms the AIC for autoregressive processes up to order two. Lardic and Mignon (2004) perform a Monte Carlo study of model selection criteria when the exact maximum likelihood method is used to estimate ARFIMA models. They limit their study to $\operatorname{ARFIMA}(0, d, 0)$ and to $\operatorname{ARFIMA}(1, d, 0)$. They also find that the SIC outperforms the AIC. To my knowledge, no results are available concerning the performance of model selection criteria for long memory models with $p$ greater than two and including moving average terms (i.e. $q \neq 0$ ). Moreover, the information criteria studied are usually limited to the commonly used AIC and SIC criteria.

Exploratory analysis on the presence of fractional integration in the agricultural spot and futures markets has shown that inference on the presence or absence of long memory in an autoregressive and fractionally integrated moving average model $[\operatorname{ARFIMA}(p, d, q)]$ is highly dependent on the number of lags included in the shortterm dynamics, i.e. the level of $p$ and $q$. This is complicated by the fact that this exploratory analysis finds that the standard information criteria disagree on where to cut off the lags, with the Akaike Information Criterion typically selecting up to ten 
lags, far more than the Schwartz Information Criterion. ${ }^{1}$

This paper hopes to contribute to the existing research on order and model selection for $\operatorname{ARFIMA}(p, d, q)$ processes. My first contribution is that I perform a Monte Carlo experiment on the performance of model selection criteria for $\operatorname{ARFIMA}(p, d, q)$ models with higher order autoregressive and moving average terms. More specifically, I let $d$ range from -0.5 to +0.5 and $p$ and $q$ range from zero to eight. In my exploratory analysis, I noticed that the AIC typically selects orders of $p$ and $q$ up to ten, while the SIC selects lower order models. The inclusion of models up to order eight should thus give an indication of the impact of complicated models on the quality of order selection and on the ability of the information criteria to make correct inferences on the presence or absence of fractional integration in the data.

My second contribution consists of extending the model selection criteria to include not only the commonly used AIC and SIC criteria but also the multimodel inference approach (MMI) discussed by Burnham and Anderson (2002) and Claeskens and Hjort (2003). When testing the significance of an estimator, the data and model are generally taken as given. However, in real life applications, the true data generating process is usually unknown. The AIC and the SIC are tools to select the best model among a set of candidates models. Inference is then conditional on the selected model. When the selected model is clearly superior to the other models considered, we can be relatively confident that inference based on this model is adequate. Unfortunately,

\footnotetext{
${ }^{1}$ In the previous paragraphs, the terms order versus model selection can lead to some confusion. When I use the term 'order selection', I mean the selection of the number of lags of the short-term dynamics of the ARFIMA $(p, d, q)$ model. Thus, order selection refers to the level of $p$ and $q$ selected. When I use the term 'model selection' or 'inference', we refer to the significance of the fractional integration parameter $d$. The term 'model selection' distinguishes between ARFIMA and ARMA models.
} 
it is more common that no model in the set under consideration is superior to the others. In that case, inference conditional on the best model can lead to serious biases, especially if the estimated values of the fractional integration parameter $d$ vary considerably across the models. To solve the above problem, the multimodel inference approach uses information obtained from all candidate models analyzed.

My third contribution is that I use four sets of candidate models in my tests for order selection and for inference. In the first test, the true data generating process is included in the set of candidate models. In the second test, the true data generating process is excluded. In the third test, all processes where the order of the autoregressive and moving average terms is equal to that of the true data generating process are excluded. In the fourth test, all processes where the order of the autoregressive and moving average terms is equal to that of the true data generating process, plus or minus one, are excluded. This addresses the concern of Burnham and Anderson (2002) that Monte Carlo studies, which generally include the true data generating process in the set of candidate models, give a biased view on the performance of model selection criteria. In real life, the true data generating process is unknown and is unlikely to be included in the set of candidate models. Moreover, we cannot rule out the possibility that the set of candidate models is relatively distant from the truth. At best, we can hope to find the best approximating model to make meaningful inferences on the parameters of interest.

The rest of the paper is organized as follows. Section 2 briefly reviews the theory on autoregressive and fractionally integrated moving average processes and on model selection. The methodology used for empirical estimation and testing is presented. Section 3 discusses the design and the results of the Monte Carlo experiments. Finally, Section 4 concludes. 


\section{Model}

\subsection{The ARFIMA model}

The scope of this paper is limited to one-step $\operatorname{ARFIMA}(p, d, q)$ type models. There are many other ways to test for fractional integration. The most popular alternative tests are the Geweke-Porter-Hudak test (1983) and the Gaussian semiparametric methods. These tests are two-step procedures: in the first step, the long-term component is estimated while in the second step, the short-term dynamics are added. Following Lardic and Mignon (2004), I prefer the use of the one-step $\operatorname{ARFIMA}(p, d, q)$ model because, in a two-step approach, the estimate of the long-term component can be contaminated by the short-term dynamics present in the data.

The ARFIMA $(p, d, q)$ model introduced by Granger and Joyeux (1980), Granger (1980, 1981) and Hosking (1981) can be written as:

$$
\Phi(L)(1-L)^{d}\left(y_{t}-\mu\right)=\Theta(L) \varepsilon_{t},
$$

where $L$ denotes the backshift operator such that $L y_{t}=y_{t-1} ; d$ is the fractional integration parameter; $\Phi(L)=\sum_{j=0}^{p} \phi_{j} L^{j}$ and $\Theta(L)=\sum_{j=0}^{q} \theta_{j} L^{j}$ are polynomials with $\phi_{0}=1, \theta_{0}=1$ and roots outside the unit circle; $\mu$ is the mean and $\varepsilon_{t}$ is white noise. The process is covariance stationary for $-0.5<d<0.5$ and mean reverting

for $d<1$. As shown by Hosking, the fractional differencing parameter is defined by the binomial series expansion for any $d>-1$ :

$(1-L)^{d}=\sum_{j=0}^{\infty}(-1)^{j}\left(\begin{array}{l}d \\ j\end{array}\right)(L)^{j}=1-d L+\frac{d(d-1)}{2 !} L^{2}-\frac{d(d-1)(d-2)}{3 !} L^{3}+\ldots$

The $\operatorname{ARFIMA}(p, d, q)$ model is estimated using the modified profile likelihood estimation specified by An and Bloomfield (1993) to reduce the bias of the exact maximum likelihood estimator in the presence of unknown nuisance parameters in the regres- 
sors. The ARFIMA model is estimated using the Ox package for ARFIMA of Doornik and Ooms (2001). We refer to their documentation for the details of the estimation procedure.

\subsection{Information criteria}

The Akaike Information Criterion (AIC) was defined by Akaike (1973) as

$$
A I C=-2 \log \mathscr{L}+2 K
$$

where $\log \mathscr{L}$ is the $\log$-likelihood of the estimated model and $K$ is the number of estimated regression parameters. It is based on the Kullback-Leibner (KL) distance, defined for continuous functions as the integral:

$$
I(f, g)=\int f(x) \log \left(\frac{f(x)}{g(x \mid \theta)}\right) d x,
$$

where $I(f, g)$ denotes the information lost when $g$ is used to approximate $f$. Unfortunately, to use the KL-distance, one must know the true model, which is exactly what we want to find. It was Akaike's merit to find an information criterion that is equivalent to the KL-distance, but independent of the true model. It can be shown that the AIC is an asymptotically unbiased estimator of the KL-distance.

The Schwartz Information Criterion (SIC) was defined by Schwartz (1978) in a Bayesian context as

$$
S I C=-2 \log \mathscr{L}+K \log (n)
$$

where $\log \mathscr{L}$ is the $\log$-likelihood of the estimated model, $K$ is the number of estimated regression parameters and $n$ is the sample size. It assumes that a true model exists and is included in the set of candidate models under consideration. The criterion provides a consistent estimator of the order or dimension of the true model. The AIC and the SIC differ from each other in that the first is an estimate of the KL-distance while the second is not related to the KL-distance. In many time-series 
simulation studies, the SIC outperforms the AIC in selecting the right order of the model.

The multimodel inference approach (MMI) is designed for situations where there is no clearly superior model in the set of candidate models. When that happens, inference based on the 'best' model is risky and mostly incorrect. Burnham and Anderson (2002) propose to base inference on model-averaged parameter estimates to use the information from the full set (or a meaningful subset) of models. In the MMI approach, the models in the set of candidate models are ranked and weighted according to their AIC or SIC score with respect to the other models in the set. For each model $i$ in the set of candidate models $(1,2, \ldots, R)$, the fractional integration parameter $d_{i}$ is estimated along with its estimated standard errors $\sqrt{\widehat{v a r}\left(\hat{d}_{i}\right)}$. The MMI estimate of $\hat{\bar{d}}$, the model averaged estimate of the fractional integration parameter, is given by

$$
\hat{\bar{d}}=\sum_{i=1}^{R} w_{i} \hat{d}_{i}
$$

and the model averaged variance estimators are defined as

$$
\widehat{\operatorname{var}}(\hat{\bar{d}})=\left[\sum_{i=1}^{R} w_{i} \sqrt{\widehat{\operatorname{var}}\left(\hat{d}_{i}\right)+\left(\hat{d}_{i}-\hat{\bar{d}}\right)^{2}}\right]^{2}
$$

where $w_{i}$ is defined as the Akaike or the Schwartz weights,

$$
w_{i}=\frac{\exp \left(-\frac{1}{2} \Delta_{i}\right)}{\sum_{r=1}^{R} \exp \left(-\frac{1}{2} \Delta_{r}\right)}
$$

with either $\Delta_{i}=A I C_{\text {best }}-A I C_{i}$ or $\Delta_{i}=S I C_{\text {best }}-S I C_{i}$. 
Table 1: Coefficients of the long-memory filter $(1-L)^{d}$ as implemented for estimation

\begin{tabular}{|r||rrrrr|rr|}
\hline$d$ & $l a g=50$ & $l a g=100$ & $l a g=150$ & $l a g=200$ & $l a g=250$ & $l a g$ & coef \\
\hline-0.4 & 0.0430113 & 0.0284110 & 0.0222846 & 0.0187555 & 0.0164072 & 500 & 0.0097753 \\
-0.2 & 0.0095112 & 0.0054671 & 0.0039537 & 0.0031413 & 0.0026279 & 500 & 0.0015096 \\
0.2 & -0.0015750 & -0.0006847 & -0.0004208 & -0.0002979 & -0.0002279 & 496 & -0.0001001 \\
0.4 & -0.0011298 & -0.0004269 & -0.0002418 & -0.0001615 & -0.0001182 & 281 & 0.0001003 \\
\hline
\end{tabular}

Note: Columns 2 to 6 show the coefficient values for the long-memory filter polynomial $(1-L)^{d}$ for the associated lags. Column 7 shows the truncation lag as implemented for estimation and column 8 shows the coefficient value at the truncation lag.

\section{Monte Carlo Simulations}

\subsection{Experimental design}

\subsubsection{Data Generating processes}

In my Monte Carlo investigation, I generate data based on the model described in Equation (1), more specifically:

$$
\Phi(L)(1-L)^{d}\left(y_{t}-\mu\right)=\Theta(L) \varepsilon_{t}
$$

for $d$ equal to $\{-0.4,-0.2,0.2,0.4\}$ and $p$ and $q$ equal to $\{0,1,2,4,6,8\}$. Thus, I examine 144 different ARFIMA specifications. To generate the data, the polynomial in Equation (2) has to be expanded. Following the method of Bhardwaj and Swanson (2006), the polynomial is truncated when the coefficients become smaller than $1.0 e-004$ for positive values of $d$. For negative values of $d$, I have chosen a lag truncation of $500 .^{2}$ Table 1 reports the values of the coefficients associated with different lags in the expansion of $(1-L)^{d}{ }^{3}$ All observations are generated using standard normal errors.

\footnotetext{
${ }^{2}$ Due to the negative value of $d$, the coefficients decrease at a much slower rate than for positive d's. I choose an arbitrary cutoff of 500 lags to simulate a long dependence in the data.

${ }^{3}$ The table is similar to the table in Bhardwaj and Swanson (2006) for positive values of $d$.
} 
Table 2 summarizes the data generating processes and parameters used in our experiments. All experimental results are based on sample sizes of 5001 observations and 500 replications of each model. This results in 72,000 series. For each model and each replication, we generate 10,000 observations. To avoid startup problems due to the long-memory property of the models only the last 5001 observations are used . Figure 1 shows the behavior of a sample of the simulated series for the different values of $d$.

For each model and each simulation run, I estimate the $\operatorname{ARFIMA}(p, d, q)$ process for $p$ and $q$ ranging from 0 to 10 . This means that I estimate 121 models for each of the 72,000 series simulated. For one run of 121 estimations, the computer needed 09 hours and 12 minutes of CPU time. This means that the total estimations took 656,640 hours on one CPU, or an equivalent of almost 75 years. ${ }^{4}$ To speed up the process, I was able to use the high performance computing system of the Katholieke Universiteit Leuven. It consists of 164 dual Opteron250 servers, with 2GB RAM, 123 4-way Opteron servers with 4GB RAM, several nodes with 16GB RAM, and two 8-way nodes. In total, the high performance computing system has 256 nodes that are interconnected with a fast Infiniband network.

For each of the 500 replications of the 144 series, I test the two main objectives of this paper. The first aim is to test which information criterion, the AIC or the SIC, leads to superior order selection for $\operatorname{ARFIMA}(p, d, q)$ models. To test for order selection, I compare the bias and mean squared error of the level of $p$ and $q$ selected by AIC and SIC. The second aim is to compare the performance of the AIC, the SIC and the multimodel inference approach to infer the presence, absence and level of fractional integration in the $\operatorname{ARFIMA}(p, d, q)$ models under consideration. I compare

\footnotetext{
${ }^{4} \mathrm{CPU}$ time for one run is not a constant. Another run took 09 hours and 56 minutes for example. Possibly other runs were faster. It is certain however that the estimation were very CPU-intensive.
} 
Table 2: Monte Carlo data generating processes and parameters used in experiments

\begin{tabular}{|l|rrrrrl|}
\hline \multicolumn{6}{|l|}{ Data Generating Processes } \\
\hline \hline$d_{0}$ & -0.4 & -0.2 & 0.2 & 0.4 & & \\
$p_{0}$ & 0 & 1 & 2 & 4 & 6 & 8 \\
$q_{0}$ & 0 & 1 & 2 & 4 & 6 & 8 \\
\hline \hline
\end{tabular}

\begin{tabular}{|l|rrrrrrrr|}
\hline \hline \multicolumn{7}{|c|}{ Parameters used in Monte Carlo simulations } & & \\
\hline$p_{0}$ & $\phi_{1}$ & $\phi_{2}$ & $\phi_{3}$ & $\phi_{4}$ & $\phi_{5}$ & $\phi_{6}$ & $\phi_{7}$ & $\phi_{8}$ \\
\hline \hline 1 & 0.5 & & & & & & & \\
2 & 0.3 & 0.1 & & & & & & \\
4 & 0.6 & 0.55 & -0.192 & -0.054 & & & & \\
6 & 0.3 & 0.77 & -0.051 & -0.1336 & -0.00852 & 0.00216 & & \\
8 & 0.8 & 0.86 & -0.508 & -0.2929 & 0.07046 & 0.038484 & 0.00096 & -0.000518 \\
\hline$q_{0}$ & $\theta_{1}$ & $\theta_{2}$ & $\theta_{3}$ & $\theta_{4}$ & $\theta_{5}$ & $\theta_{6}$ & $\theta_{7}$ & $\theta_{8}$ \\
\hline 1 & -0.7 & & & & & & & \\
2 & -0.4 & 0.21 & & & & & & \\
4 & 0.1 & 0.47 & -0.081 & -0.0126 & & & & \\
6 & -0.2 & 0.6 & 0.05 & -0.0839 & 0.00432 & 0.00126 & & \\
8 & -0.6 & 0.88 & 0.354 & -0.2559 & -0.04524 & 0.02984 & -0.000878 & -0.000404 \\
\hline
\end{tabular}

the bias and mean squared error of the fractional integration parameters $d$ selected using the AIC and the SIC and the model averaged estimates of the multimodel inference approach. Instead of taking the model averaged estimate of the 121 models estimated, I include estimation results for the best models only. The rationale for reducing the number of models used to compute the model averaged estimate is that most information is included in the models with highest weights. Models with low weight may affect results by adding noise even though their information content is poor. Therefore, I include models with descending Akaike and Schwartz weights until those weights sum to 0.90 . The weights are then rescaled to sum to one.

\subsubsection{Rationale and hypotheses}

The choice of the models and information criteria is based on the following considerations. 
Figure 1: Sample of simulated series
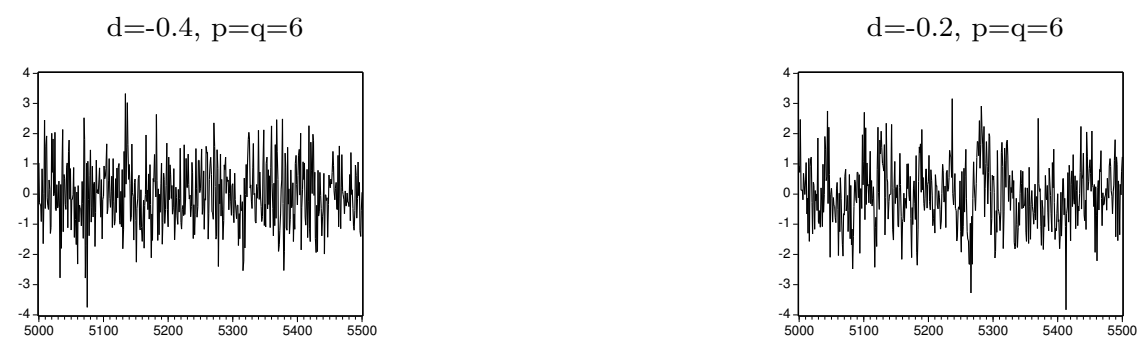

$$
\mathrm{d}=0.2, \mathrm{p}=\mathrm{q}=6
$$
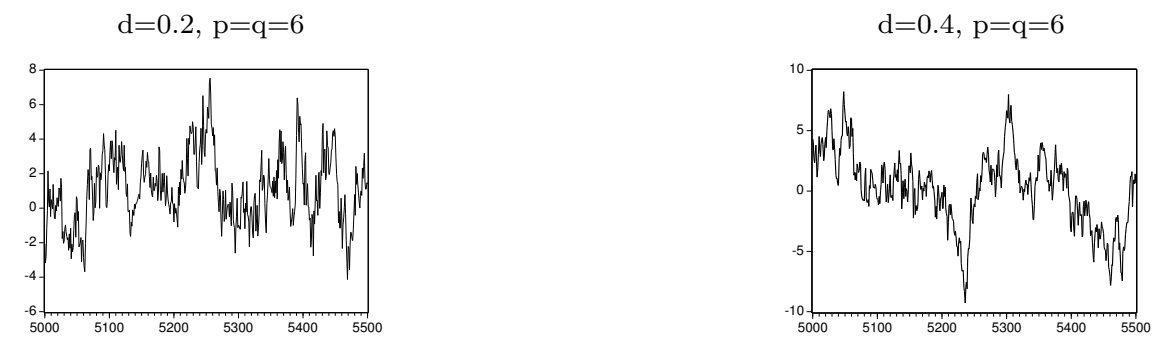

The first consideration concerns the level of $d$. I assume that $d$ is nonzero and $d$ is an element of $\left\{-\frac{1}{2}, \frac{1}{2}\right\}$. Model selection for short memory processes has been sufficiently covered in the literature to omit them here; see Bhansali (1994) for a review on stationary short-memory autoregressive processes.

The second consideration concerns the order of the data generating processes. For each $d$, I choose to let the models range from few to many autoregressive and moving average terms. This should provide insights into the quality of the model selection criterion to find the correct value of $p$ and $q$ and to estimate the value of $d$ when the short-memory process has a high order. In the literature, the consensus has emerged that the AIC overestimates the true order of the data while the SIC provides a consistent estimator of the order of the model, assumed to be low. It can be shown that the SIC will select the correct model with probability one in an infinite sample when the true data generating process is included in the set of candidate models. The simulation literature confirms this result and finds that the SIC does well even 
in moderate-sized samples. However, as discussed in Burnham and Anderson (2002), this result has been tested mostly on simple processes with few tapering effects. We test the impact of adding more autoregressive and moving average terms on the order selection quality of both information criteria. Since AIC has been shown to overestimate the true order of the data for low $p$ and $q$ and since SIC selects the model with the lowest dimension possible, I hypothesize that:

H1: SIC outperforms AIC in terms of order selection when the order of the true data generating process is low and vice versa.

The third consideration concerns my choice of model selection criteria. As discussed in Section 2, I test the performance of order selection based on the AIC and the SIC. I choose these model selection criteria because they are the most widely used in the literature. I further test the quality of the estimation of $\hat{d}$ when model selection is based on the AIC and SIC and their multimodel inference counterparts (MMAIC and MMSIC, respectively). I compare the bias and variance of $\hat{d}$ based on AIC, MMAIC, SIC and MMSIC to test whether the multimodel inference approach is worth the extra effort. Since I hypothesize that the SIC outperforms AIC in terms of order selection for low order models and vice versa, I expect the same relation to hold for inference on the presence or absence of fractional integration. I hypothesize that when the order of $p$ and $q$ are closer to the true order, the estimated value of $d$ will also be closer to the true $d$. This leads to my second hypothesis:

H2: SIC outperforms AIC in terms of inference when the order of the true data generating process is low and vice versa. 
The multimodel inference approach bases inference on model-averaged parameter estimates to use the information of a meaningful subset of models. Given that the MMAIC and MMSIC use more information than the AIC and SIC, these models might provide better results for the inference of the fractional integration parameter than their best model counterparts.

H3: MMAIC and MMSIC outperform AIC and SIC in terms of inference whatever the order of the true data generating process.

The fourth and last consideration concerns the choice of models considered in the set of candidate models. In this paper, I test the performance of the AIC and the SIC to select the correct order of the model and the performance of the AIC, the SIC and the MMI to discriminate between long and short memory models. Though most studies find that the SIC generally outperforms the AIC both in terms of order selection and inference, Burnham and Anderson (2002) question the results of these Monte Carlo experiments. The generating process is often simple and included in the set of candidate models while, with real data, the true generating process is unknown so that the true model is often not included in the set of candidate models. Thus, the goal of an information criterion is not to find the true model in a set of simple candidates models, but to find the best model to approximate the truth. I address the concern of Burnham and Anderson (2002) by performing four model selection runs for four different sets of candidate models:

Set 1: the true data generating process is included in the set of candidate models;

Set 2: the true data generating process is excluded from the set of candidate models;

Set 3: the processes with autoregressive and moving average terms equal to the true $p_{0}$ and $q_{0}$ are excluded from the set of candidate models; 
Set 4: the processes with autoregressive and moving average terms equal to $p_{0} \pm 1$ and $q_{0} \pm 1$ are excluded from the set of candidate models.

Following Burnham and Anderson (2002), I expect the performance of the SIC compared to the AIC in terms of order selection and inference to worsen when the truth is excluded from the set of candidate models. The SIC is derived to select the true model from a set of candidate models that includes the true data generating process. The AIC on the other hand is derived to minimize the KL-distance and looks for the best approximating model to make meaningful inferences on the parameters of interest, here the fractional parameter $d$. Therefore, I expect the performance of the SIC to worsen when the true data generating process and models close to the true data generating process are excluded from the set of candidate models. This leads to the final hypothesis:

H4: Compared to set 1, the performance of SIC and MMSIC as compared to AIC and MMAIC worsens in sets 2, 3 and 4 .

\subsection{Results on order selection}

The literature on model/order selection typically mentions the bias and the mean squared errors of the estimators as variables of interest to evaluate the performance of model selection criteria. The bias is defined as the mean estimation error of the estimator, here the level of $p$ and $q$ :

$$
\operatorname{Bias}(p)=E\left(\hat{p}-p_{0}\right) \quad \operatorname{Bias}(q)=E\left(\hat{q}-q_{0}\right)
$$

where $\hat{p}$ and $\hat{q}$ are the estimated values of $p$ and $q$, and $p_{0}$ and $q_{0}$ are the true $p$ and $q$ (i.e. the $p$ and $q$ of the data generating process). The mean squared error (MSE) 
is defined as the mean squared estimation error:

$$
M S E(p)=E\left(\hat{p}-p_{0}\right)^{2} \quad M S E(q)=E\left(\hat{q}-q_{0}\right)^{2} .
$$

Given that the MSE can be computed as

$$
M S E=\text { Bias }^{2}+\text { Var }
$$

the MSE actually reflects the bias and the variance of the estimation error of $p$ and $q$

Tables 3 and 4 show the bias of the estimation error of the autoregressive terms and the moving average terms respectively using AIC and SIC for the four sets of candidate models considered. 5 Tables 5 and 6 give the mean squared errors of the estimation error. Tables 13 and 14 in appendix detail the variance of the estimation error. These tables are given in appendix because the results can be deduced from the bias and MSE tables. Please note that when I talk about the estimation error of $p$ and $q$, I mean $\hat{p}-p_{0}$ where $\hat{p}$ is the estimated order of the autoregressive terms and $p_{0}$ is the order of the data generating process. I do not discuss the bias and variance of the coefficients $\phi$ and $\theta$ of the ARFIMA model. For clarity, the results in the tables are cross-tabulated with respect to the order of $p_{0}$ and $q_{0} \cdot{ }^{6}$ The following results emerge from the Monte Carlo experiment.

First, in line with the literature on AIC and SIC, the results show that, on average, the AIC overestimates the level of $p$ and $q$ while the SIC underestimates it. However,

\footnotetext{
${ }^{5}$ As a reminder: Set 1 includes the data generating process, set 2 excludes the data generating process, set 3 excludes all models with $p=p_{0}$ and $q=q_{0}$ and set 4 excludes all models with $p=p_{0} \pm 1$ and $q=q_{0} \pm 1$

${ }^{6}$ More detailed tables are available for the referee in a separate file. The tables will not be included in the final version, but will be available from the author upon request.
} 
the results show that, ceteris paribus, the bias and the MSE of $\hat{p}$ decreases with the order of the autoregressive term used in the data generating process when the AIC is used, while it increases when the SIC is used. This result is consistent across the four sets of candidate models considered. A similar finding holds for the bias and the MSE of $\hat{q}$ : it decreases with the order of the moving average term used in the data generating process when the AIC is used, but increases when the SIC is used. Note that the bias and the MSE of $\hat{p}$ and $\hat{q}$ are largely independent of the level of $q_{0}$ and $p_{0}$ respectively.

Second, it can be deduced that, as far as the bias and the MSE are concerned, the SIC clearly outperforms the AIC for values of $p_{0}$ and $q_{0}$ up to four, but that the AIC outperforms the SIC when $p_{0}$ and $q_{0}$ equal six or eight. The above provides support for hypothesis 1: the SIC seems to outperform the AIC in terms of order selection when the order of the true data generating process is low and vice versa. Note that the results are not as clear-cut for the variance of $\hat{p}$ and $\hat{q}$. On the one hand, there does not seem to be a clear pattern for the variance. On the other hand, the variance of $\hat{p}$ and $\hat{q}$ is clearly lower for all orders of the data generating process and for all sets of candidate models when the SIC is used. The MSE is clearly driven by the bias while the variance is relatively constant across lags.

Third, contrary to hypothesis four, it cannot be concluded that the quality of order selection using SIC as compared to the performance of order selection using AIC worsens when models close to the true data generating process are excluded from the set of candidate models. Instead, the performance of the AIC and the SIC worsens in a similar fashion. 


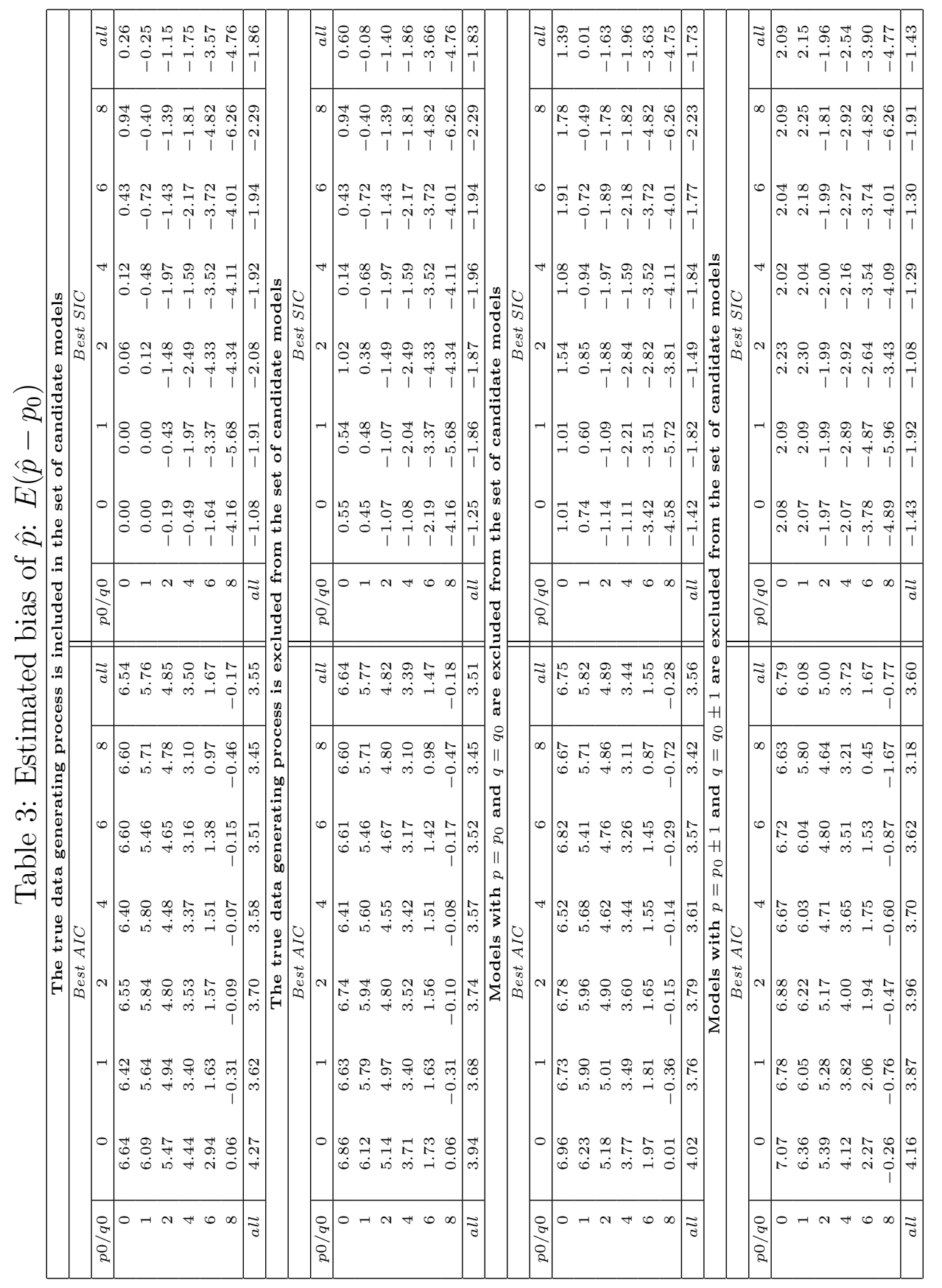




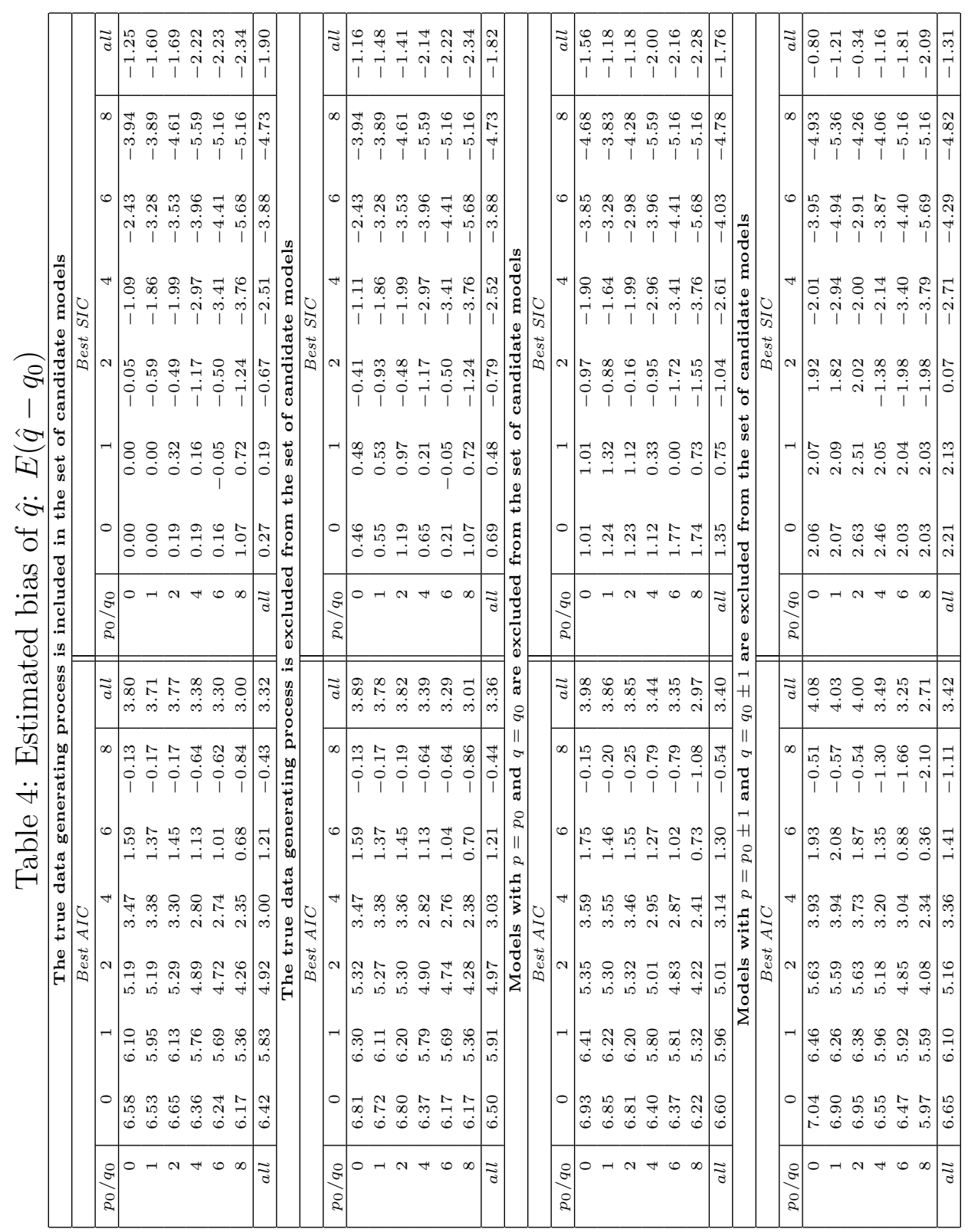




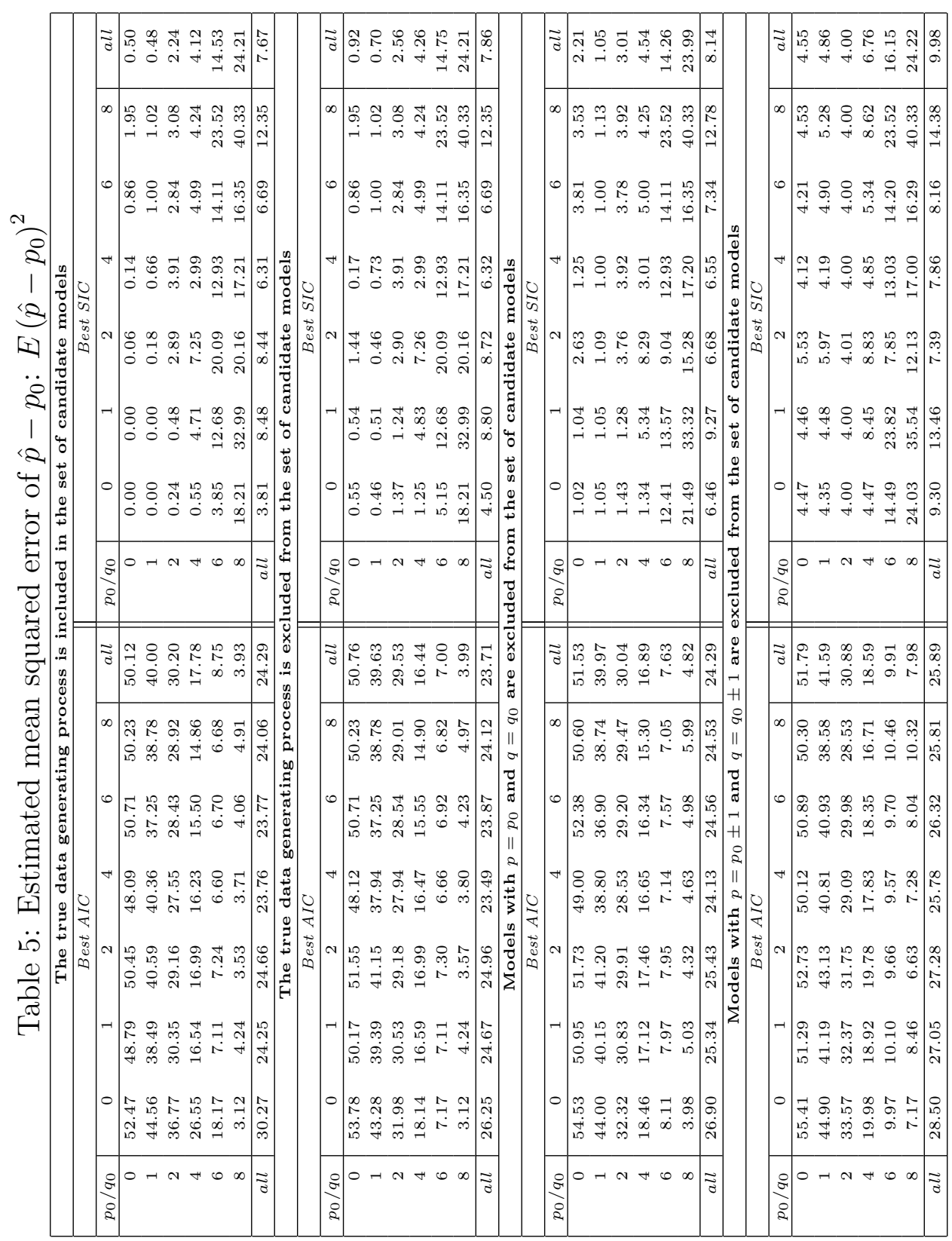




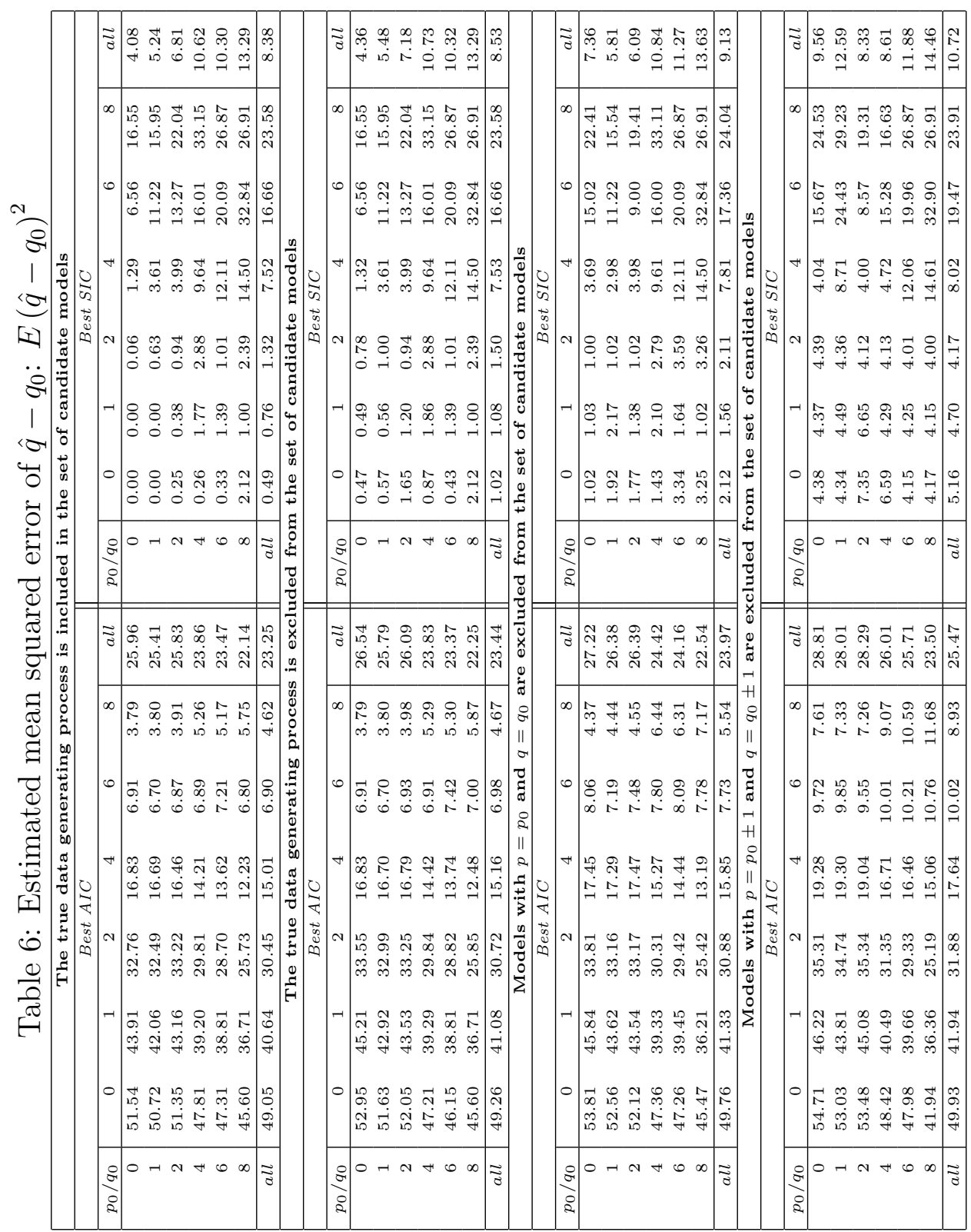




\subsection{Results on inference}

I study model selection not only as a means to select the right order of $p$ and $q$, but also as a tool to discriminate between the presence or absence of fractional integration. Therefore, I am interested in the estimated bias of $\hat{d}$, the variance of $\hat{d}$ and the probability of type-II errors (i.e. the frequency with which an information criterion rejects the presence of fractional integration in the data when the true data generating process is fractionally integrated). Tables 7 and 8 compare the estimated bias of $\hat{d}$ for the four sets of candidate models when the AIC, the SIC and their multimodel counterparts are used. Tables 9 and 10 compare the mean squared error of $\hat{d}$ when the AIC, the SIC and their multimodel counterparts are used. Tables 15 and 16 in appendix compare the variance of $\hat{d}$ when the AIC, the SIC and their multimodel counterparts are used. Tables 11 and 12 compare the probability of a type-II error for the four sets of candidate models when the AIC, the SIC and their multimodel counterparts are used. I find mixed results on the hypotheses.

First, the results of my Monte Carlo experiment contradict hypothesis two. I find that the SIC outperforms the AIC in terms of bias, MSE and variance whatever the order of the true data generating process and the set of candidate models considered. The SIC also leads to a much lower probability of a type-II error in all cases under consideration. Similar results hold for the multimodel SIC compared to the multimodel AIC.

Second, the results provide mixed evidence for the use of the multimodel inference approach and lead to a rejection of hypothesis three. While there is evidence in the data that the MMAIC outperforms the AIC in terms of inference, the SIC on the contrary dominates its multimodel counterpart whatever the order of the data generating process. Moreover, both the AIC and the SIC have a lower probability of a type-II error than their respective multimodel counterparts. 
Third, the Monte Carlo experiments also lead to a rejection of the final hypothesis: based on the results, I cannot confirm that the performance of SIC and MMSIC as compared to AIC and MMAIC worsens when the true data generating process and processes close to the true data generating process are excluded from the set of candidate models. On the contrary, the presence or absence of the data generating process in the set of candidate models does not seem to influence the choice of the model selection criterion. Thus, the critique of Burnham and Anderson (2002) does not seem to be applicable in this situation.

The results allow an additional comment on model selection for fractionally integrated models. The higher the order of the autoregressive terms, the more imprecise $\hat{d}$ is estimated. This result is consistent across sets of candidate models and model selection criteria. It is also confirmed by the probability of a type-II error. The presence of many tapering effects clearly has an impact on the estimation quality. This result supports the use of a one-step procedure instead of a two-step procedure to test for the presence of fractional integration. 


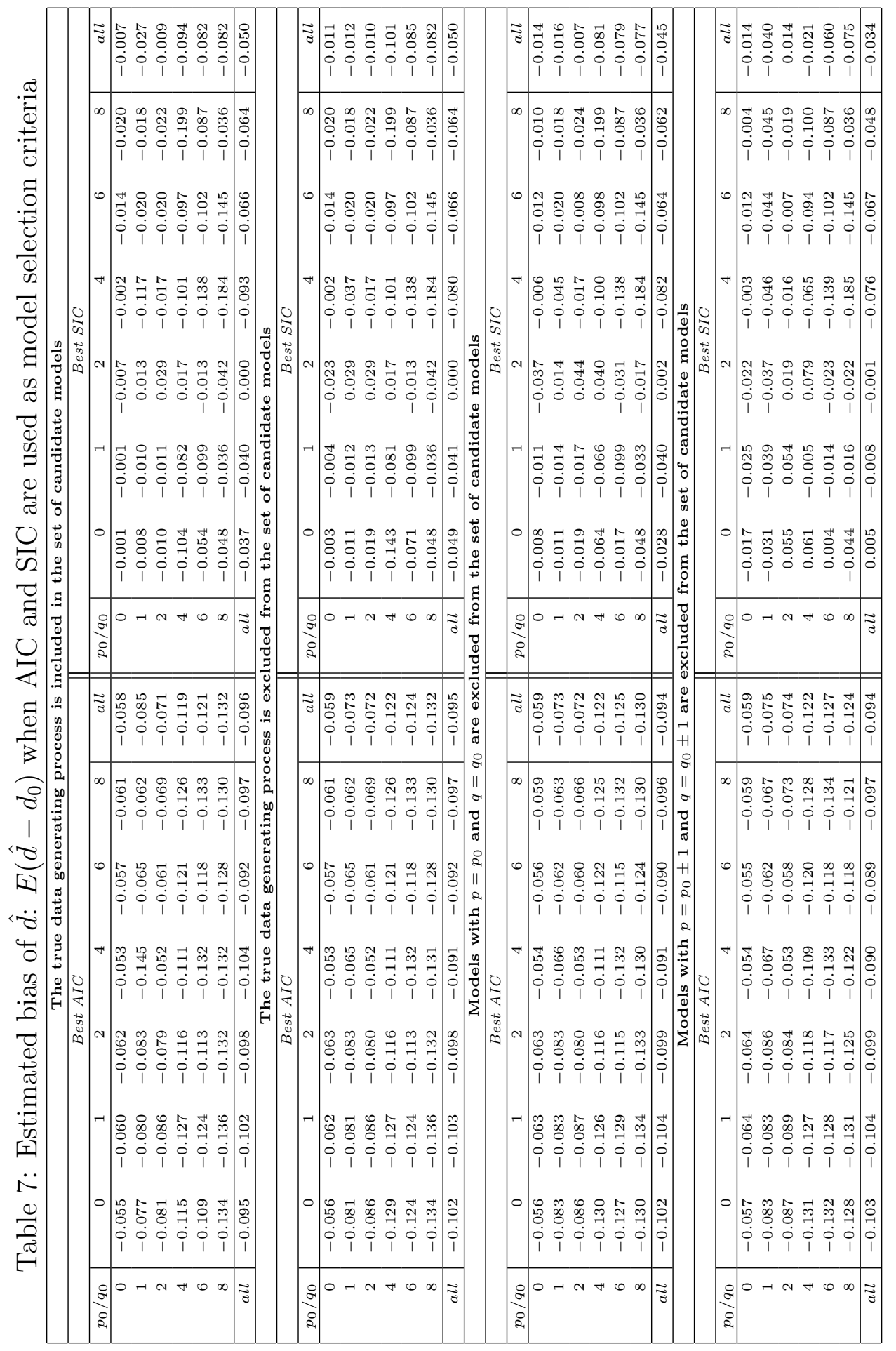




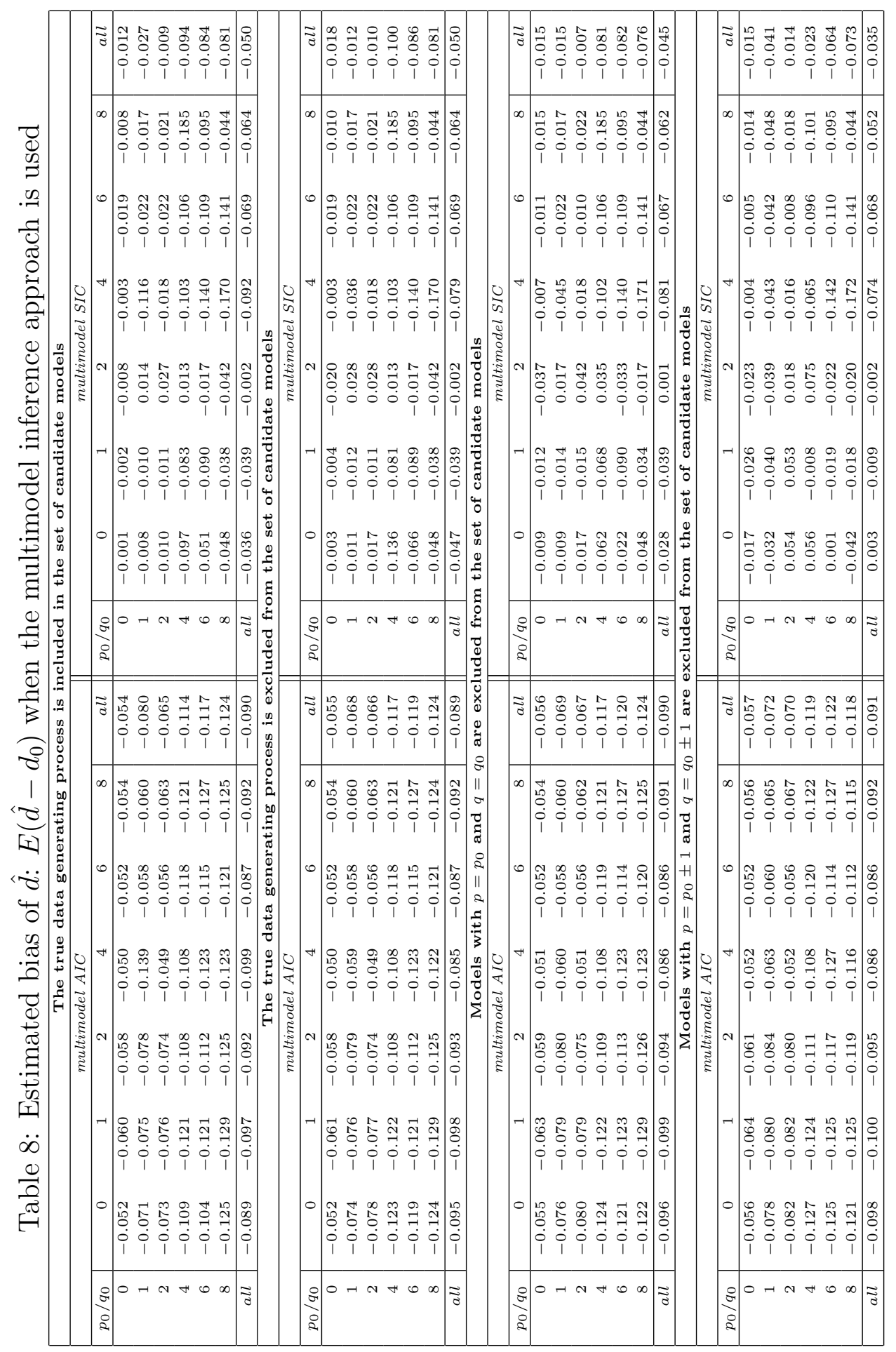




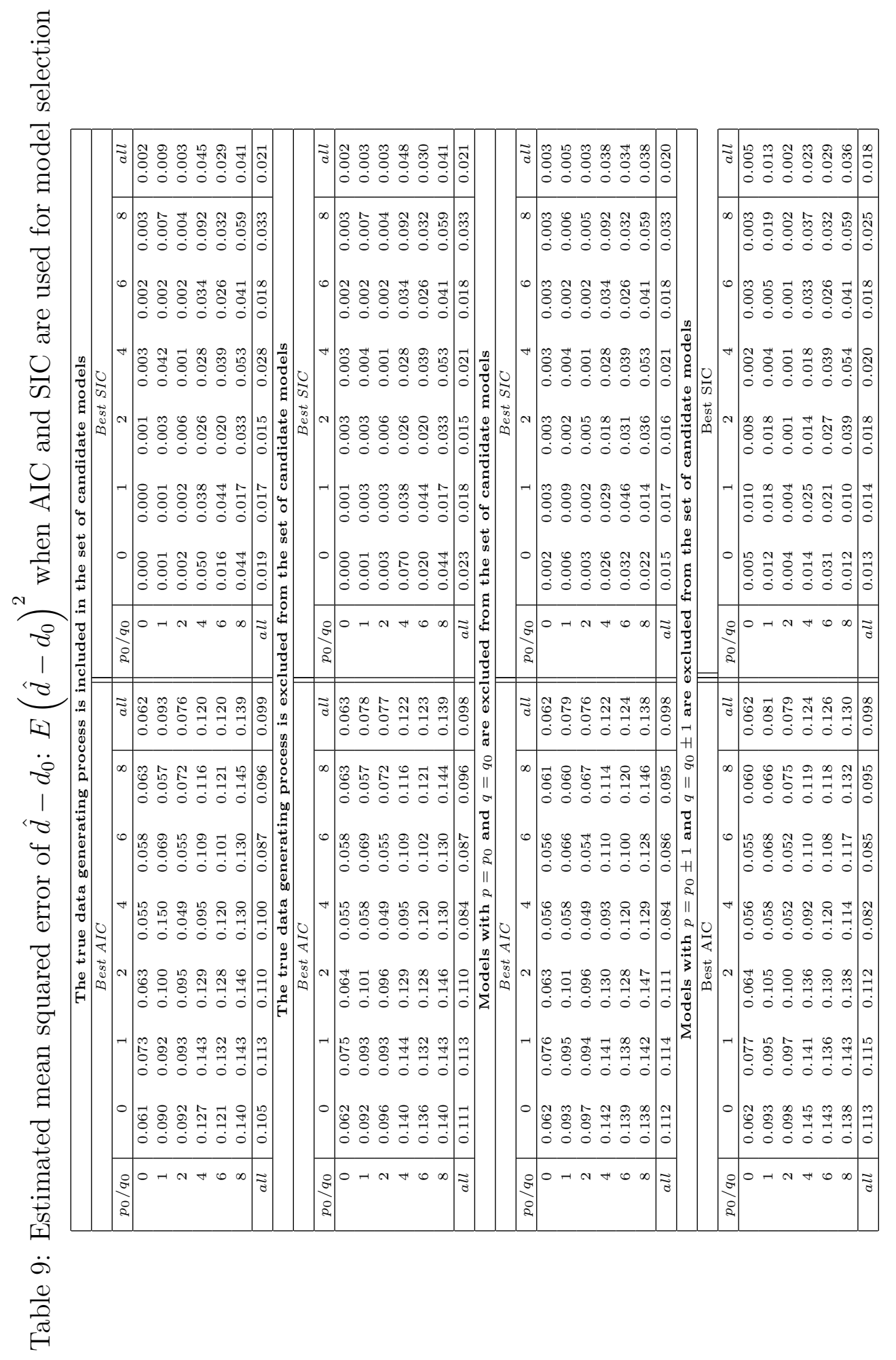




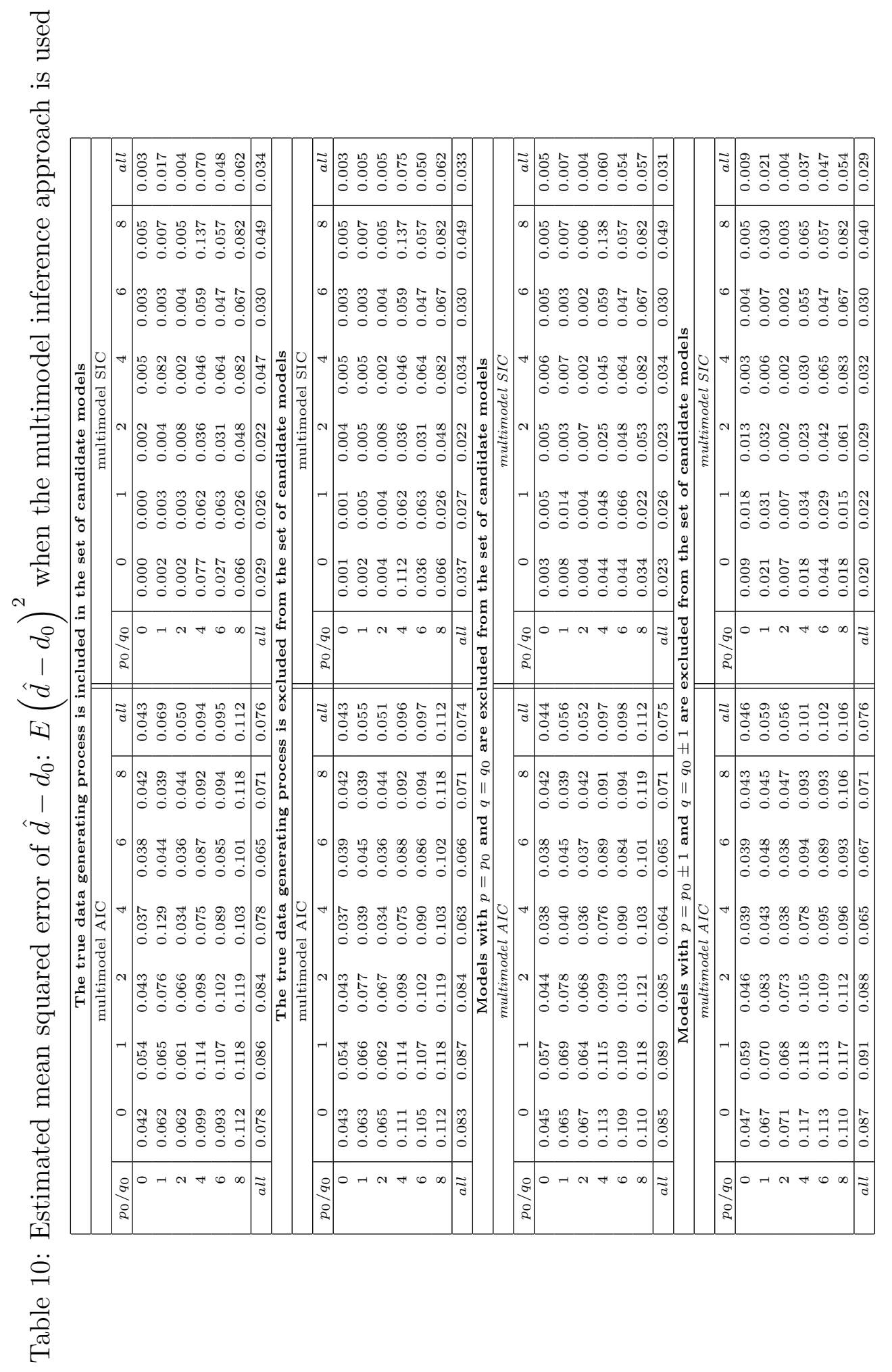




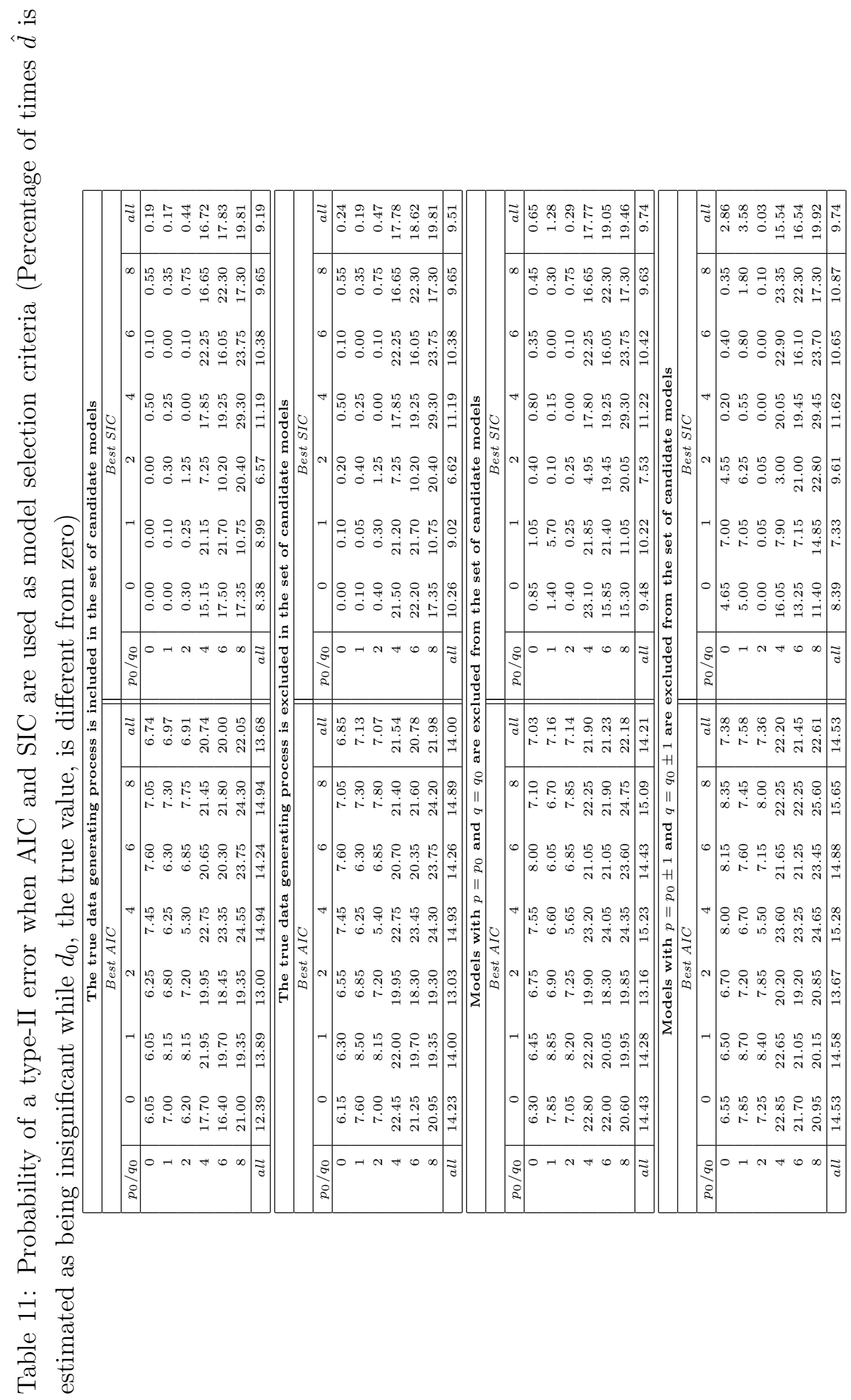




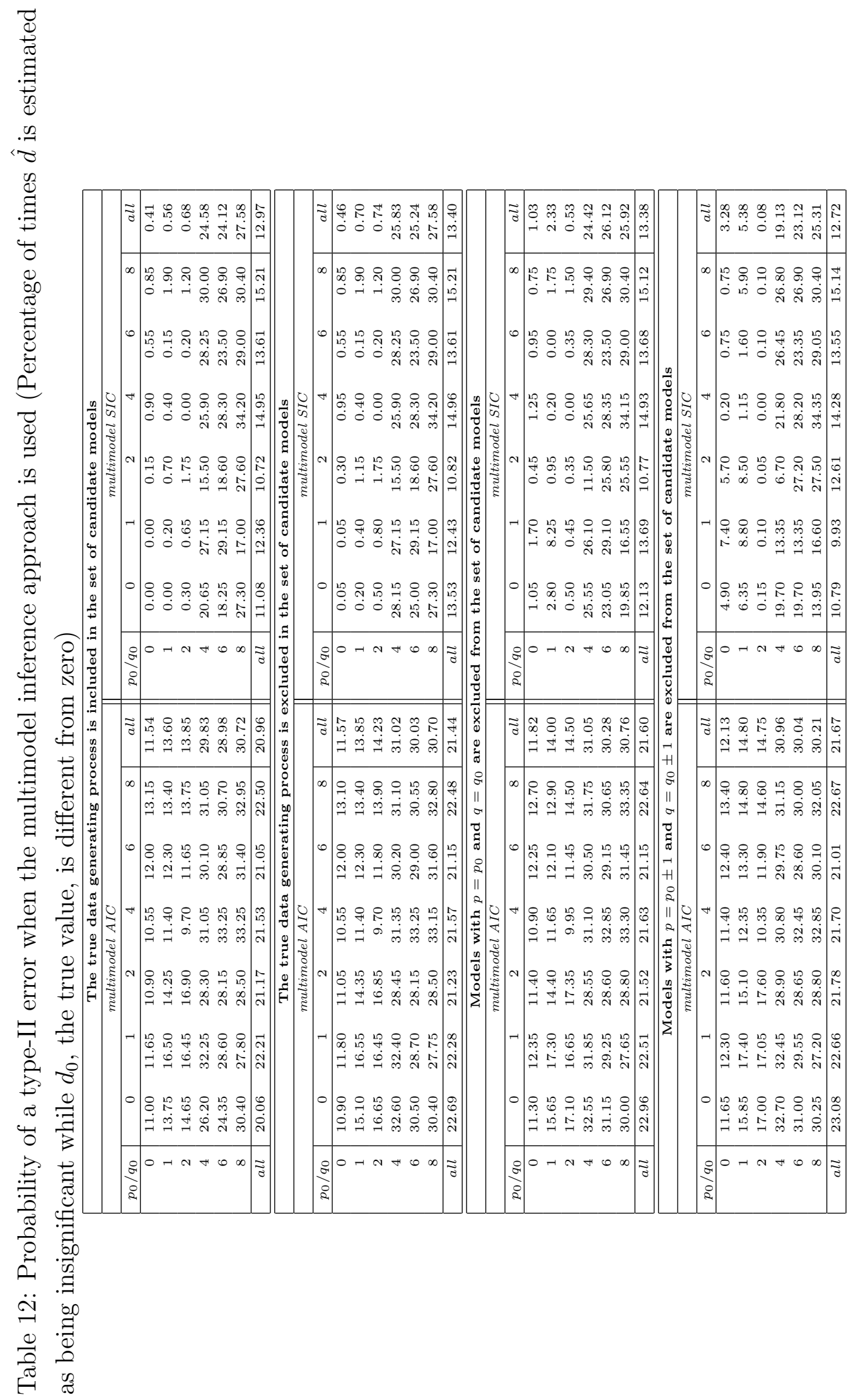




\section{Conclusion}

In this paper, I perform an extensive Monte Carlo study to analyze the performance of the $\mathrm{AIC}$ and the SIC to select the number of lags of the autoregressive and moving average terms of an $\operatorname{ARFIMA}(p, d, q)$ model and the performance of the AIC, the SIC and their multimodel counterparts to make meaningful inferences on the fractional integration parameter $d$. Additionally, I analyze the impact of including many lags in the data generating process on order selection and inference quality. Finally, I test whether the inclusion of the data generating process in the set of candidate models has an influence on the choice of the information criteria. The following conclusions emerge from my study. For order selection, as expected, the SIC clearly outperforms the AIC in terms of bias and MSE when the number of lags is smaller than four while the AIC outperforms the SIC when the number of lags is six or eight. However, the SIC leads to a smaller variance of the estimation error of $p$ and $q$ whatever the order of the data generating process. This result is consistent across all sets of candidate models considered. Thus, the choice of AIC versus SIC for order selection should be conditioned on prior beliefs/knowledge concerning the number of lags contained in real-world data. For inference on the fractional integration parameter, the SIC outperforms the AIC and the multimodel information criteria in terms of bias, MSE and variance. This result is consistent across all sets of candidate models considered and for all types of data generating processes. Though the number of lags is often better approximated by the AIC, inference on the fractional integration parameter is better when fewer lags are included in the model. In this context, the choice of an information criterion seems to depend on the purpose the model has to fulfill: order selection versus inference on the presence or absence of fractional integration. 


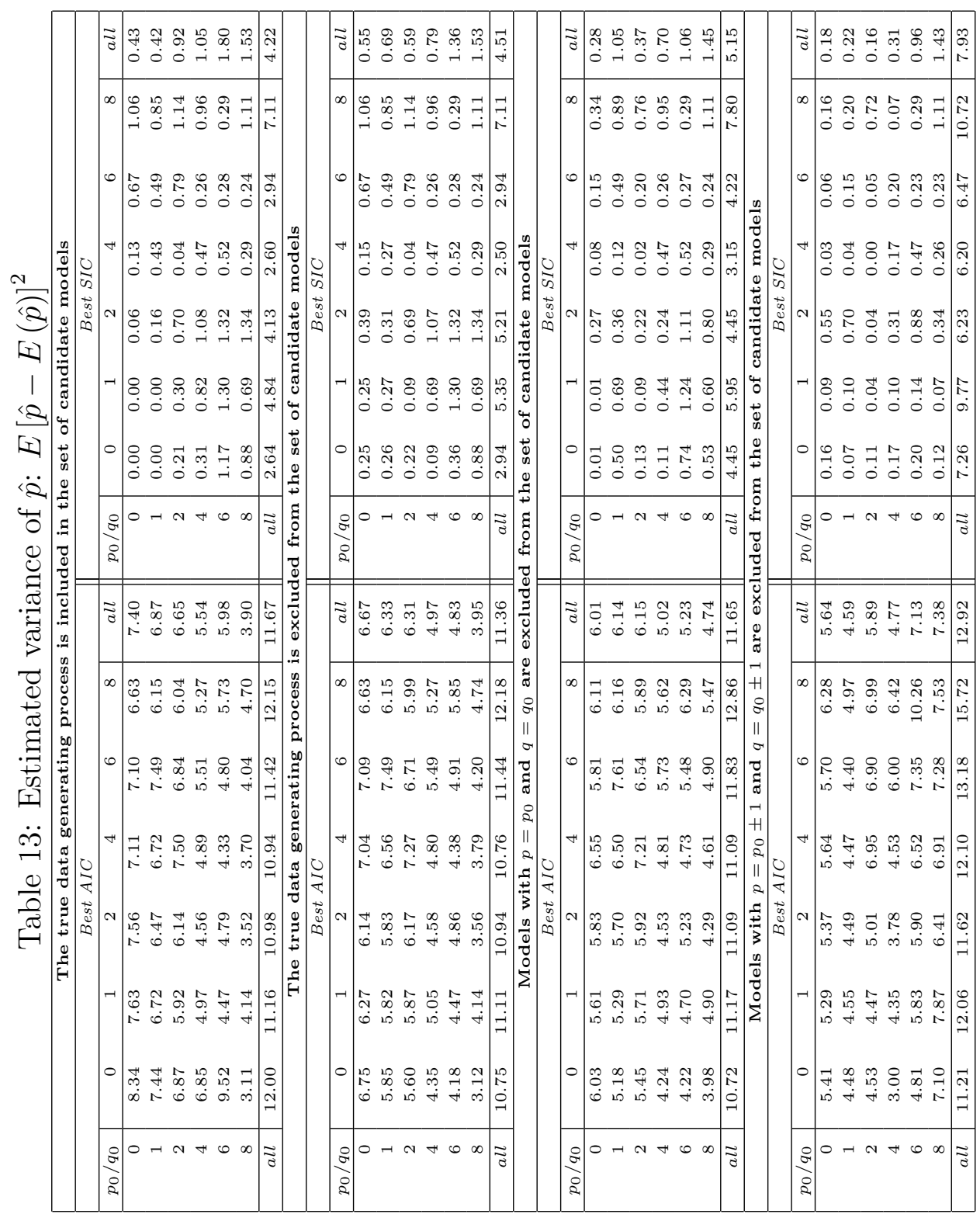




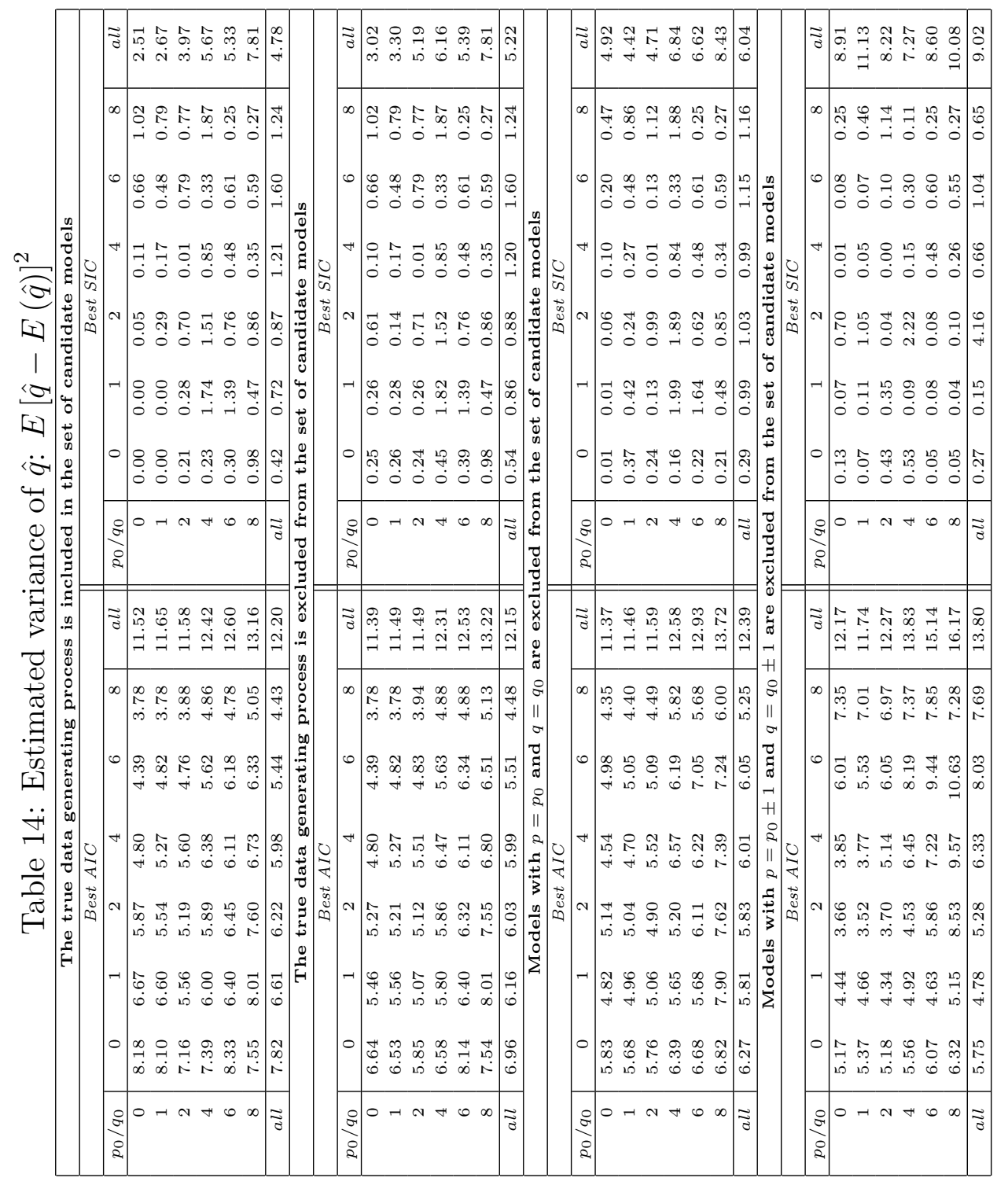




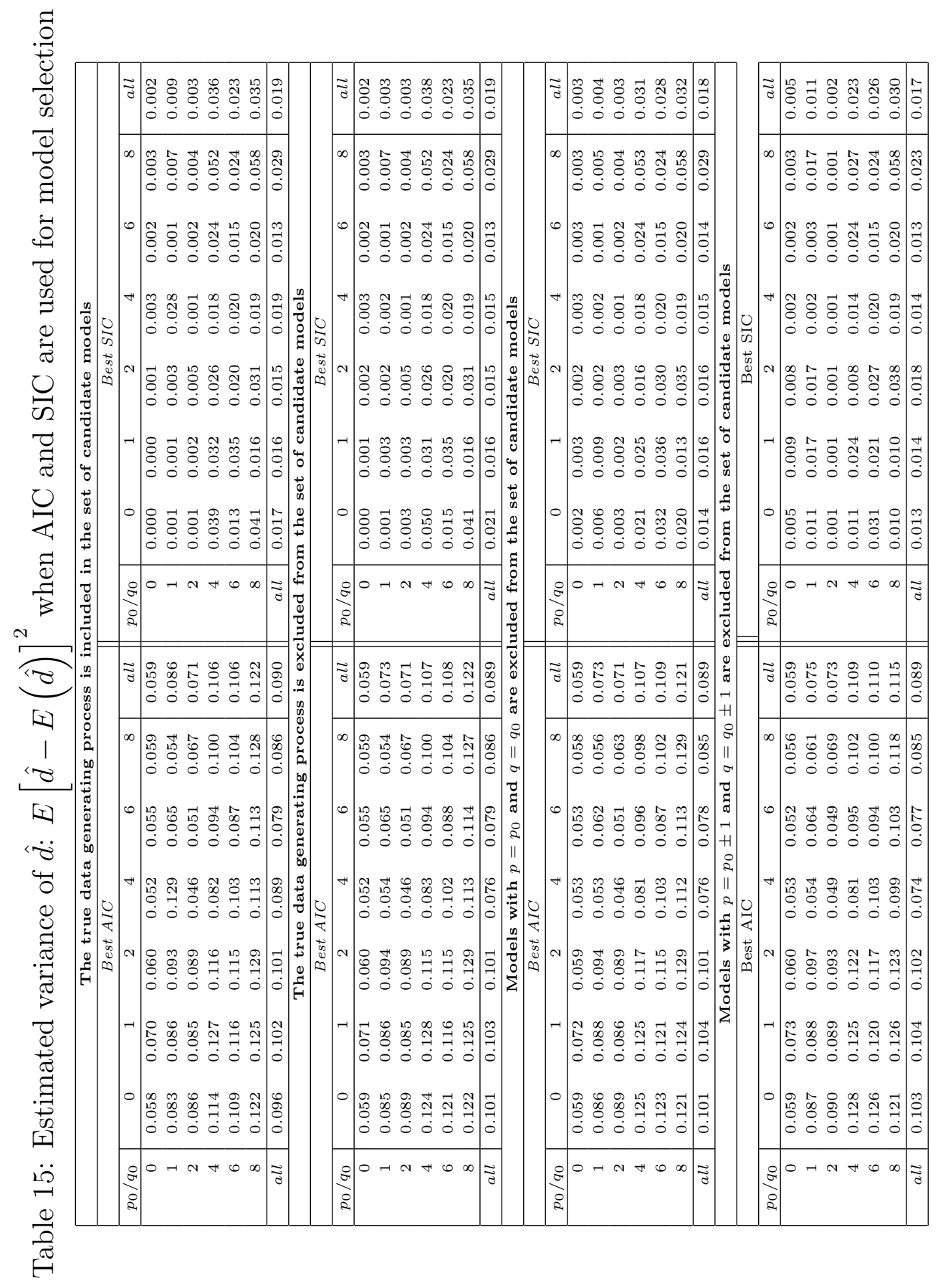




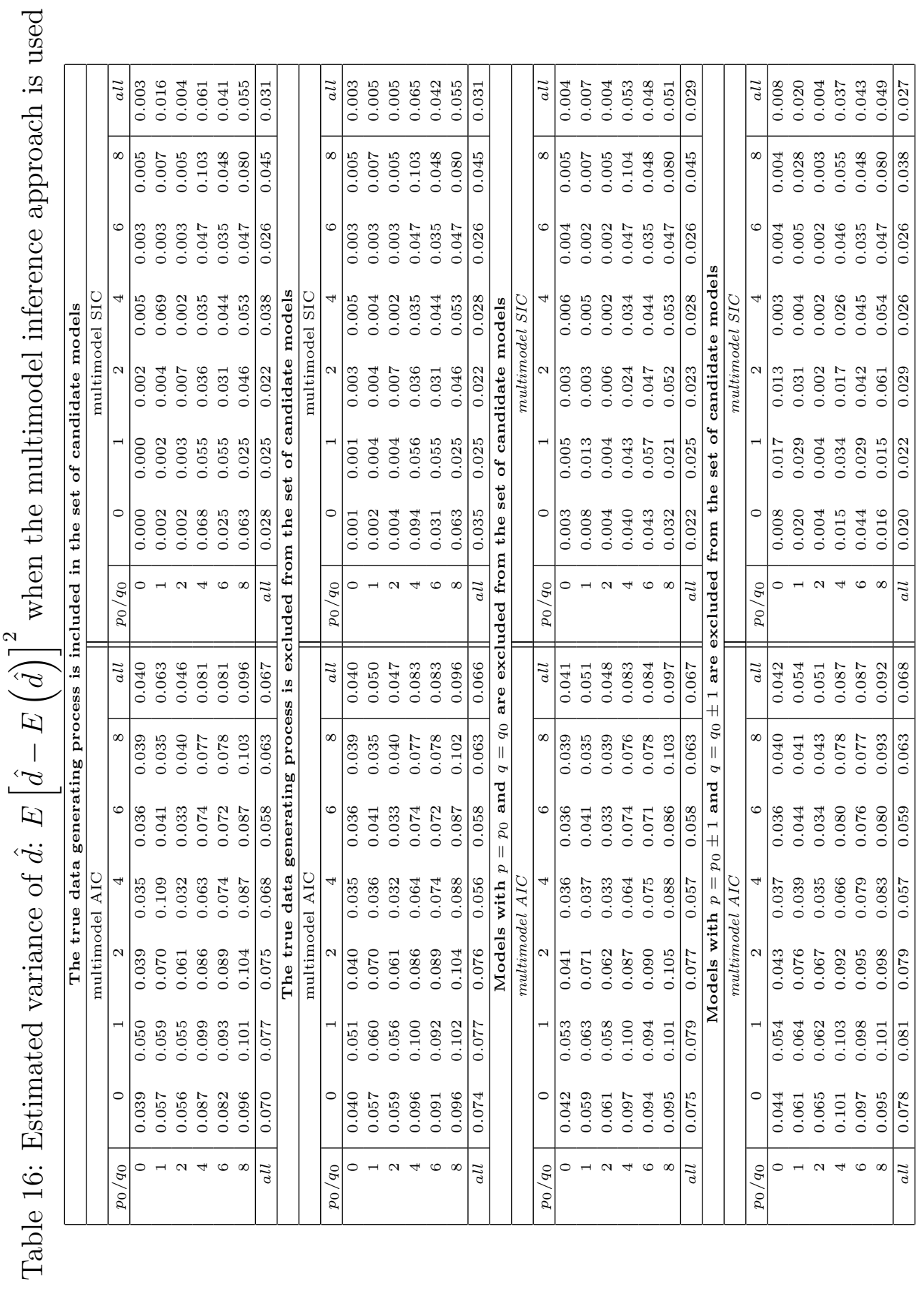




\section{References}

[1] Baillie, R.T. (1996) 'Long memory processes and fractional integration in econometrics', Journal of Econometrics, Vol. 73, pp. 5-59.

[2] Beran, J., Bhansali, R.J. and Ocker, D. (1998) 'On unified model selection for stationary and nonstationary short- and long-memory autoregressive processes', Biometrika, Vol. 85, pp. 921-934.

[3] Bhardwaj, G. and Swanson, N.R. (2006) 'An empirical investigation of the usefulness of ARFIMA models for predicting macroeconomic and financial time series', Journal of Econometrics, Vol. 131, pp. 539-578.

[4] Bollerslev, T. and Mikkelsen, H.O. (1996) 'Modeling and pricing long memory in stock market volatility', Journal of Econometrics, Vol. 73, pp. 151-184.

[5] Burnham, K.P. and Anderson, D.R. (2002) Model selection and multimodel inference: a practical information-theoretic approach. Springer-Verlag, New York.

[6] Claeskens, G. and Hjort, N.L. (2003) 'Frequentist model average estimators', Journal of the American Statistical Association, Vol. 98, pp. 879-899.

[7] Geweke, J. and Porter-Hudak, S. (1983) 'The estimation and application of long memory time series models', Journal of Time Series Analysis, Vol. 4, pp. 221-238.

[8] Granger, C.W.J. (1980) 'Long memory relationships and the aggregation of dynamic models', Journal of Econometrics, Vol. 14, pp. 227-238.

[9] Granger, C.W.J. (1981) 'Some properties of time series data and their use in econometric model specification', Journal of Econometrics, Vol. 16, pp. 121-130.

[10] Hurst, H.E. (1951) 'Long-term storage capacity of reservoirs', Transactions of the american society of civil engineers, Vol. 116, pp. 770-199.

[11] Jin, H.J. and Frechette, D.L. (2004) 'Fractional integration in agricultural futures price volatilities', American Journal of Agricultural Economics, Vol. 86, pp. $432-$ 443.

[12] Lardic, S. and Mignon, V. (2004) 'The exact maximum likelihood estimation of ARFIMA processes and model selection criteria: a monte carlo study', Economics Bulletin, Vol. 3, pp. 1-16.

[13] Lo, A.W. (1991) 'Long term memory in stock market prices', Econometrica, Vol. 59, pp. 1279-1313.

[14] Ooms, M. and Doornik, J.A. (1998) Estimation, simulation and forecasting for fractional autoregressive integrated moving average models, Discussion Paper, Econometric Institute, Erasmus University Rotterdam, presented at the fourth annual meeting of the Society for Computational Economics, June 30, 1998, Cambridge, UK.

[15] Robinson, P. (2003) Time series with long memory. Oxford University Press, Oxford.

[16] Sowell, F.B. (1992a) 'Maximum likelihood estimation of stationary univariate fractionally integrated time series models', Journal of Econometrics, Vol. 53, pp. 165-188. 
[17] Sowell, F.B. (1992b) 'Modeling long run behavior with the fractional ARIMA model', Journal of Monetary Economics, Vol. 29, pp. 277-302. 